existing client) (adjusted (AOR) 2.66, 95\% CI 2.08 to 3.44), speaking a Mandarin or Cantonese language (AOR 3.01, 95\% CI 1.67 to 5.42), inconsistent condom use for vaginal sex at work (AOR 12.54, 95\% CI 7.32 to 21.48), and being older than 40 years (AOR 2.85, 95\% CI 1.91 to 4.25). Thai language speakers were less likely to report inconsistent condom use for fellatio (AOR 0.44 , 95\% CI 0.23 to 0.83). No significant association was demonstrated for injection drug use or sexual practice outside of work. During the study period 17 of the 1539 sex workers (1.1\%, 95\% CI 0.6 to 1.8) were diagnosed with pharyngeal gonorrhoea.

Conclusions These finding suggest interventions to promote condoms for fellatio by sex workers are needed. We could not determine if inconsistent condom use for fellatio at work was directly associated with pharyngeal gonorrhoea due to the low sample size of cases. Further research into the determinants of this behaviour, particularly among different language groups is warranted.

\section{P1-S2.20 FACTORS INFLUENCING CERVICAL INFECTION AMIONG FEMALE SEX WORKERS IN BENIN}

doi:10.1136/sextrans-2011-050108.77

${ }^{1} \mathrm{~S}$ Diabaté, ${ }^{1} \mathrm{~A}$ Chamberland, ${ }^{2} \mathrm{~N}$ Géraldo, ${ }^{3} \mathrm{M}$ Zannou, ${ }^{4} \mathrm{M}$ Massinga-Loembé, ${ }^{3} \mathrm{~S}$ Anagonou, ${ }^{5} \mathrm{~A}$ Clabbé, ${ }^{1} \mathrm{C}$ Tremblay, ${ }^{6} \mathrm{M}$ Alary. ${ }^{1}$ Centre de recherche du Centre hospitalier de I'Université de Montréal, Montréal, Canada; ${ }^{2}$ Dispensaire IST, Centre de santé de Cotonou I, Cotonou, Benin; ${ }^{3}$ Centre national hospitalier universitaire, Cotonou, Benin; ${ }^{4}$ Institut de Médecine tropicale, Anvers, Belgium; ${ }^{5}$ Université de Montréal, Hôpital Maisonneuve-Rosemont, Montréal, Canada; ${ }^{6} U R E S P$, Centre de recherche FRSO du CHA universitaire de Québec, Québec, Canada

Background As female sex workers (FSW) continue to play a key role in HIV-1 epidemic in sub-Saharan Africa, programs aiming at reducing sexually transmitted infections (STI) involved in HIV acquisition and transmission are still needed. The objective of this study was to determine factors influencing STI acquisition among FSW in Cotonou, Benin.

Methods Three hundred and eighty seven (387) FSW were recruited in a medical center dedicated to STI treatment among female sex workers and their clients. Cervical infections by Neisseria gonorrhoeae (NG) and Chlamydia trachomatis (CT; SDA, BD Probe Tec ${ }^{\circledR}$ ) were investigated at enrolment into the study. All infected FSW received adequate treatment for free. We used a multivariate logistic regression model to assess variables independently associated with cervical infection at enrolment ( $p$ value $<0.05$ ).

Results The FSW were essentially from Benin (39.5\%) and neighbouring countries such as Nigeria (22.1\%), Togo $(21.8 \%)$ and Ghana $(15.1 \%)$. One hundred and forty three subjects $(143 ; 37.0 \%)$ were HIV-1 positive (52 were treated with antiretroviral therapy and 91 remained untreated because not yet meeting eligibility for treatment). Median age (IOR) and median duration (IOR) in the work were $33(27-40)$ and $3(1.2-5)$ years. Cervical infections by NG and CT were diagnosed in $4.4 \%$ and $3.9 \%$ cases, respectively. Only two women $(0.5 \%)$ had both infections. Seventy seven per cent $(77.1 \%)$ and $86.8 \%$ of the FSW reported, respectively, consistent condom use during the last 7 days of work and condom use at last sexual intercourse with a client. After adjustment for age and condom use, being HIV-1 infected [RR 3.04; 95\% CI 1.25-to 7.40], not working in a brothel (RR 3.28; 95\% CI 1.64-to 9.27), and being working for less than 12 months (RR 3.55; 95\% CI 1.53-to 8.23) were independently associated with cervical infection in our study population

Conclusion Our results suggest that HIV-1 infection and cervical infection by NG and CT alter each other. Antiretroviral therapy in association with preventive programmes aiming at controlling STI should be encouraged among new FSW and also among those working outside brothels.

\section{P1-S2.21 INTEGRATING PUBLIC HEALTH INTO THE SEX WORKER COMMUNITY IN INDIANAPOLIS}

doi:10.1136/sextrans-2011-050108.78

${ }^{1} \mathrm{~A}$ M Roth, ${ }^{1} \mathrm{~J} \mathrm{G}$ Rosenberger, ${ }^{2} \mathrm{~J}$ Waters, ${ }^{2} \mathrm{~J}$ Arno, ${ }^{1} \mathrm{~B}$ Van Der Pol. ${ }^{1}$ Indiana University, USA; ${ }^{2}$ Marion County Health Department, Indianapolis, USA

Background Syphilis is a significant disease from a public health perspective both historically and as a result of the serious consequences of infection. While studies have explored the epidemiology of syphilis among at-risk populations, limited information exists regarding women's preferences for STD testing and treatment services. The purpose of this study was to explore sex workers preferences for receiving sexual health services.

Methods A total of six groups of 4-7 sex workers each were recruited from local community-based organisations. The semistructured interview guide included questions based on the Health Belief Model such as -1) cues to action-"Think back to the last time you received a female wellness exam or got tested for STD. What prompted you to schedule that appointment?" 2) Perceived susceptibility- "Describe what worries you most when you think about your health and how it's affected by being on the streets." and 3) perceived barriers - "What are some of the reasons why women who are working the streets don't get tested for STD?" Focus groups lasted $1 \mathrm{~h}$, were audio recorded, and transcribed. Preliminary codes were selected, applied to the transcripts, and key concepts that spanned groups were identified.

Results In general, most women used utilised health protective behaviours to mitigate health risks associated with sex work. However, these behaviours were context and partner specific. Participants were interested in having increased access to sexual health services. Primary themes regarding program development included-experiences with healthcare providers, types of services that would encourage uptake, mechanisms for service delivery and the importance of collaborating with law enforcement and other service providers. The participant's recommendations were organised into a model program with specific service delivery mechanisms for each key concept.

Conclusions These data suggest potential pathways STD control programs to intervene with hard-to-reach populations, like commercial sex workers. In this project, a participant-informed approach was utilised that should result in increased uptake and utilisation of sexual health services because participants provided key insights into the programmatic considerations necessary for STD prevention efforts to be most effective.

\section{P1-S2.22 CHANGES IN HIV KNOWLEDGE \& RISK BEHAVIOURS IN FEMALE SEX WORKERS IN RWANDA - HIV PREVENTION IMPLICATIONS OF TREND ANALYSES IN BEHAVIOURAL SURVEILLANCE}

doi:10.1136/sextrans-2011-050108.79

${ }^{1} \mathrm{C}$ Kayitesi, ${ }^{1} \mathrm{R}$ Hinda, ${ }^{1} \mathrm{~A}$ Gwiza, ${ }^{1} \mathrm{H}$ Balisanga, ${ }^{1} \mathrm{~S}$ Nsanzimana, ${ }^{2} \mathrm{~A}$ Koleros, ${ }^{3} \mathrm{~J}$ M V Uwimana, ${ }^{1} \mathrm{M}$ Mwumvaneza. ${ }^{1}$ TRAC Plus, Kigali, Rwanda; ${ }^{2}$ Futures Group International/MEASURE Evaluation, Kigali, Rwanda; ${ }^{3}$ Rwanda National Reference Laboratory, Kigali, Rwanda

In Rwanda, existing empirical and analytical studies indicate that female commercial sex workers (FCSW) play an important role in the HIV epidemic. It is thus critical to track sexual risk behaviours in this group in order to develop effective prevention programs and services for sex workers. In this context, the Ministry of Health $(\mathrm{MOH})$ of Rwanda and partners conducted two rounds of behavioural surveillance surveys (BSS) among FCSW in 2006 and 2010. We used time-location sampling based on geographic maps of sex work 
hotspots in Rwanda to recruit a nationally representative sample of 1040 FCSW in 2006 and 1338 FCSW in 2010. At the time of the interview, all FCSW present at the site were asked to participate in the study and administered a quantitative survey instrument after obtaining informed consent. Data were double-entered and analysed using EpiInfo, SPSS and STATA. $\chi^{2}$ Tests of significance were performed on key variables. Logistic regression analysis was performed to control for key socio-demographics. Overall, an increase in comprehensive HIV knowledge from 18\% in 2006 to $22 \%$ in 2010 was observed ( $p$ Value $<0.0001$ ). However, some misconceptions still persist, with a decline in FCSW who rejected that mosquito bites can transmit HIV from $74 \%$ in 2006 to $69 \%$ in 2010 ( $p$ value $<0.0001$ ). The median age of respondents' first paid sexual act remained the same, with a reported median age of 19 years in both surveys. The proportion of FCSW who reported having consistently used condoms in the month prior to the survey rose from $28 \%$ in 2006 to $35 \%$ in 2010 ( $p$ value $<0.001$ ). At the same time, the proportion of FCSW who reported having used a condom during their last sexual act with a paying client dropped from $84 \%$ in 2006 to $80 \%$ in 2010 ( $\mathrm{p}$ value $<0.001$ ). Comparing key indicators from the 2006 and 2010 BSS provides important insights for HIV program planners. Although the comparison indicates that overall HIV knowledge has generally improved in the time between the two surveys, some misconceptions on HIV transmission still persist. Equally, regarding sexual behaviour and practices, although consistent condom use reportedly improved between the two BSS, the overall level of reported use is still relatively low and some important indicators-including condom use at last sex with a paying client-declined. These data further underscore the importance of reaching FCSW with prevention commodities and appropriate HIV education and behaviour change communication messages.

\section{Epidemiology poster session 2: Population: transgender}

\section{P1-S2.23 TRANSGENDER PERSONS IN GUATEMALA - OVER- EXPOSED AND UNDER-PROTECTED - THE FINDINGS OF AN RDS BEHAVIOURAL SURVEY}

doi:10.1136/sextrans-2011-050108.80

${ }^{1}$ W Miller, ${ }^{1} \mathrm{~B}$ Alvarez, ${ }^{2} \mathrm{~S}$ Boyce, ${ }^{2} \mathrm{~A}$ Alvarado, ${ }^{3} \mathrm{C}$ Barrington, ${ }^{2} \mathrm{G}$ Paz-Bailey. ${ }^{1}$ Del Valle

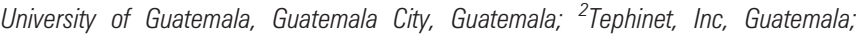
${ }^{3}$ University of North Carolina, , Chapel Hill, USA

Background Male-to-female transgender persons experience the highest HIV prevalence rates of any high risk population in Central America. The purpose of this study is to compare HIV-related risk behaviours, knowledge and exposure to prevention activities among transgender persons and other men who have sex with men (MSM) in Guatemala City.

Methods From September-to December 2010, we conducted a probability-based survey of MSM, including male-to-female transgender persons who have sex with men, using respondent-driven sampling (RDS) in Guatemala City. Data on sexual and drug use behaviour, knowledge of HIV, attitudes, stigma, access to prevention, care, and treatment services were collected by interviewers using Personal Digital Assistants (PDA). Population proportions and 95\% CIs, adjusted for RDS sampling weights, were calculated using the Respondent Driven Sampling Analysis Tool (version 6.0). Crude summary statistics were calculated in SAS (version 9.1).

Results A total of 509 MSM, including 99 male-to-female transgender persons enrolled in the study. The mean age for transgendered persons was 29.1 vs 28.0 for non-transgender MSM. Education level and socio-economic status were comparable between the two groups. Transgender persons, in comparison to non-transgender MSM, had higher rates of transactional sex $(77.6 \%$ vs $41.9 \%$ in the past year, respectively), drug use $(26.7 \%$ vs $3.3 \%$ cocaine use in the past month) and prior HIV testing (95.7\% vs $68.9 \%$, during lifetime). On the other hand, transgender persons have similar levels of forced sex (14.1\% vs $6.5 \%$ in the past year), exposure to HIV educational activities $(74.2 \%$ to $67.8 \%$ in the past year), access to free condoms (83.9\% to $79.6 \%)$ and correct knowledge of HIV prevention (54.8\% vs $41.7 \%$ ) see Abstract P1-S2.23 Figure 1. Conclusions-Compared to other MSM, transgender persons in Guatemala City more often receive money for sex and use cocaine and are at disproportionately higher risk for HIV infection. While prevention activities, such as HIV testing, seem to be reaching transgender persons, exposure may not translate into increased protection. Findings emphasise the need for innovative HIV prevention activities to address the many facets and levels of vulnerability and social marginalisation experienced by transgender persons in Guatemala City. Such efforts should extend beyond individual-level outreach and information provision to rigorously evaluated intervention strategies at the social and structural levels.

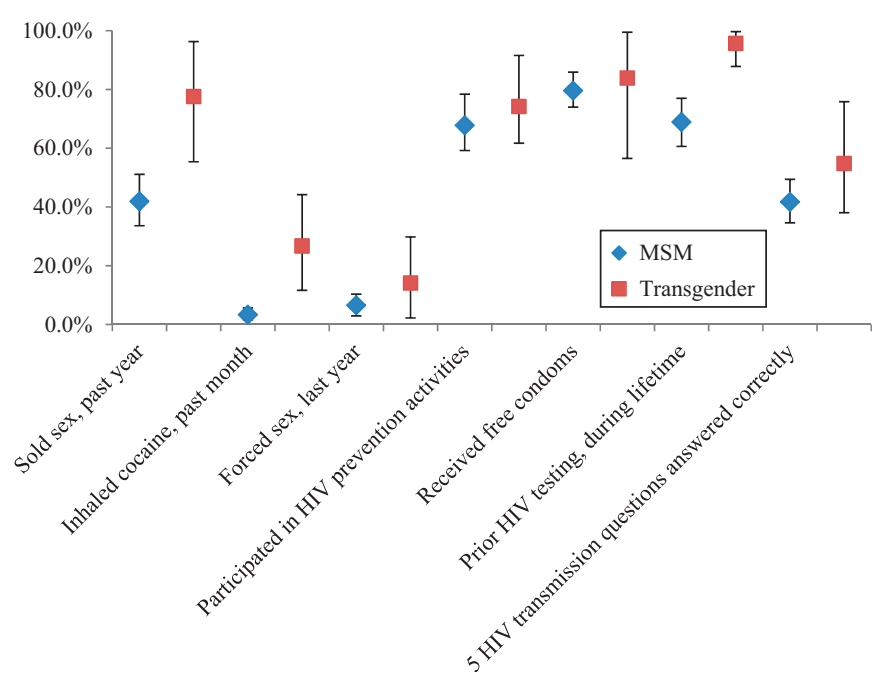

Abstract P1-S2.23 Figure 1 Behavioural and HIV Prevention Indicators. Non-transgender MSM and Transgender Persons, Guatemala City, 2010.

\section{Epidemiology poster session 2: Population: Injection drug users \\ P1-S2.24 HIV AND HEPATITIS C RISK FACTORS, INFECTION STATUS AWARENESS AND ACCESS TO CARE AMONG PEOPLE WHO INJECT DRUGS - RESULTS FROM A NATIONAL SURVEILLANCE SYSTEM, I-TRACK PHASE 2 (2005-2008)}

doi:10.1136/sextrans-2011-050108.81

J Tarasuk, A M Frescura, S Ogunnaike-Cooke, C Archibald. Public Health Agency of Canada, Ottawa, Canada

Background HIV and hepatitis C (HCV) risk factors, infection status awareness and access to care among people who inject drugs (IDU) were compared across infection groups in a national enhanced surveillance sample.

Methods Phase 2 data collection for I-Track (Enhanced surveillance of HIV and HCV risk behaviours among IDU) was conducted from 2005 to 2008 in 10 Canada sites. Confidential face-to-face interviews collected data on demographics, drug-use and sexual risk behaviours, and HIV and HCV testing. Dried blood or oral fluid samples were collected for HIV and HCV antibody testing. Descriptive statistics for demographics, risk behaviours and access to care and treatment 